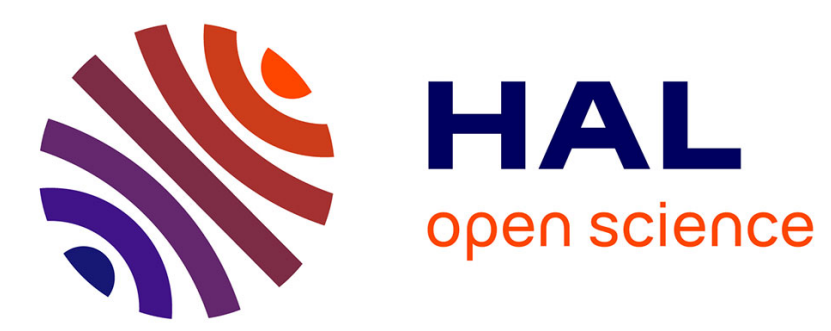

\title{
REFRACTION AND REFLEXION ACCORDING TO THE PRINCIPLE OF GENERAL COVARIANCE
}

Patrick Iglesias-Zemmour

\section{To cite this version:}

Patrick Iglesias-Zemmour. REFRACTION AND REFLEXION ACCORDING TO THE PRINCIPLE

OF GENERAL COVARIANCE. Journal of Geometry and Physics, 2019. hal-02401456

\section{HAL Id: hal-02401456 \\ https://hal.science/hal-02401456}

Submitted on 10 Dec 2019

HAL is a multi-disciplinary open access archive for the deposit and dissemination of scientific research documents, whether they are published or not. The documents may come from teaching and research institutions in France or abroad, or from public or private research centers.
L'archive ouverte pluridisciplinaire HAL, est destinée au dépôt et à la diffusion de documents scientifiques de niveau recherche, publiés ou non, émanant des établissements d'enseignement et de recherche français ou étrangers, des laboratoires publics ou privés. 


\title{
REFRACTION AND REFLEXION ACCORDING TO THE PRINCIPLE OF GENERAL COVARIANCE
}

\author{
PATRICK IGLESIAS-ZEMMOUR
}

\begin{abstract}
We show how the principle of general covariance introduced by Souriau in smoothly uniform contexts, can be extended to singular situations, considering the group of diffeomorphisms preserving the singular locus. As a proof of concept, we shall see how we get again this way, the laws of reflection and refraction in geometric optics, applying an extended general covariance principle to Riemannian metrics, discontinuous along a hypersurface.
\end{abstract}

\section{INTRODUCTION}

In his paper "Modèle de particule à spin dans le champ électromagnétique et gravitationnel” published in 1974 [Sou74], Jean-Marie Souriau suggests a precise mathematical interpretation of the principle of General Relativity. He names it the Principle of General Covariance. Considering only gravitation field ${ }^{1}$, he claimed that any material presence in the universe is characterized by a covector defined on the quotient of the set of the Pseudo-Riemannian metrics on space-time, by the group of diffeomorphisms. This principle being, according to Souriau, the correct statement of the Einsteins's principle of invariance with respect to any change of coordinates. Actually, Souriau's general covariance principle can be regarded as the active version of Einstein invariance statement, where change of coordinates are interpreted from the active point of view as the action of the group of diffeomorphisms.

Now, for reasons relative to the behavior at infinity and results requirement, the group of diffeomorphisms of space-time is reduced to the subgroup of compact supported diffeomorphisms.

Date: April 6, 2019.

1991 Mathematics Subject Classification. 83C10, 78A05, 37J10.

Key words and phrases. General Covariance, Geometric Optics, Symplectic Geometry.

${ }^{1}$ In his paper Souriau extends also his consideration to electromagnetic field. 
In consequence, after some identifications, Souriau interprets a material presence for a Pseudo-Riemannian metric $g$ on the space-time $\mathrm{M}$, responding to this principle of covariance, as a tensor distribution $\mathscr{T}$ whose test functions are compacted supported covariant 2-tensors $\delta g$ defined on $M$, which vanishes along the infinitesimal action of the compact supported diffeomorphisms group Diff ${ }_{\text {cpct }}(\mathrm{M})$. That is, in Souriau's notations:

$$
\mathscr{T}\left(\delta_{\mathrm{L}} g\right)=0
$$

where $\delta_{\mathrm{L}} g$ is the Lie derivative of the metric $g$ by any compact supported vector field $\delta x$. In a more standard notation, denoting by $\xi$ the compact supported vector field on $\mathrm{M}, \delta_{\mathrm{L}} g=£_{\xi}(g)$. A tensor distribution satisfying that last equation has been called by Souriau, an Eulerian distribution.

Among others, there are two main examples of application of this principle. The first one is a continuous distribution of matter described by a $C^{1}$-smooth symmetric contravariant 2-tensor $\mathrm{T}^{\mu \nu}$,

$$
\mathscr{T}(\delta g)=\frac{1}{2} \int_{\mathrm{M}} \mathrm{T}^{\mu \nu} \delta g_{\mu \nu} \text { vol, }
$$

where vol is the Riemannian volume associated with $g$, and we use the Einstein's convention on (up-down) repeated indices. We recall that the Lie derivative of a metric $g$ by a vector field $\xi$ is given, in terms of coordinates, by

$$
\delta_{L} g_{\mu, \nu}=\hat{\partial}_{\mu} \xi_{\nu}+\hat{\partial}_{\nu} \xi_{\mu}
$$

where $\hat{\partial}$ denotes the covariant derivative with respect with the Levi-Civita connection relative to $g$ (sometimes denoted also by $\nabla$ ). Then, the Eulerian condition above writes now:

$$
\frac{1}{2} \int_{M} \mathrm{~T}^{\mu \nu}\left(\hat{\partial}_{\mu} \xi_{\nu}+\hat{\partial}_{\nu} \xi_{\mu}\right) \operatorname{vol}=0
$$

By reason of symmetries and integrating by parts, recalling that $\xi$ is compact supported, one gets:

$$
\int_{M} \hat{\partial}_{\mu}\left(\mathrm{T}^{\mu \nu}\right) \xi_{\nu} \text { vol }=0,
$$

for all compact supported vector field $\xi$. Therefore the Eulerian condition writes:

$$
\hat{\partial}_{\mu}\left(\mathrm{T}^{\mu \nu}\right)=0, \quad \text { or } \quad \hat{\operatorname{div}}(\mathrm{T})=0,
$$

where $\hat{\operatorname{div}}(\mathrm{T})$ is the Riemannian divergence of the tensor T. Hence, the Eulerian character of the distribution $\mathscr{T}$ translates simply in the Euler conservation 
equations of the energy-momentum tensor. Which is the first principle in General Relativity. And that justifies a posteriori the chosen vocabulary.

The second example is a preamble to the subject of our paper. It concerns the construction of geodesics as Eulerian distributions supported by a curve. So, let $t \mapsto \mathrm{T}^{\mu \nu}$ be a $C^{2}$-smooth curve in the fiber bundle of symmetric contravariant 2-tensor over $\mathrm{M}$, over a curve $\gamma: t \mapsto x$. Let then,

$$
\mathscr{T}(\delta g)=\frac{1}{2} \int_{-\infty}^{+\infty} \mathrm{T}^{\mu \nu} \delta g_{\mu \nu} d t .
$$

After some astute manipulation [Sou74], Souriau shows that this distribution is Eulerian if and only if the supporting curve $\gamma$ is a geodesic, that is,

$$
\frac{\hat{d}}{d t} \frac{d x}{d t}=0
$$

And then:

$$
\mathscr{T}(\delta g)=\frac{k}{2} \int_{-\infty}^{+\infty} \frac{d x^{\mu}}{d t} \frac{d x^{\nu}}{d t} \delta g_{\mu \nu} d t
$$

where $k$ can be any real constant. In general $k$ is interpreted as the mass $m$ of the particle moving along the geodesic $\gamma$ with velocity $\mathbf{v}=d x / d t$. But for classical geometric optics $k$ will be interpreted as the color coefficient $h \nu / c^{2}$, where $b$ is the Planck constant, $\nu$ is the frequency of the ray and $c$ is the speed of light in vacuum.

Thus, the condition to be geodesic is interpreted through this approach as an immediate consequence of the principle of General Relativity, which is quite satisfactory.

This Souriau's construction has been also commented in a great way, and applied unexpectedly by Shlomo Sternbeg, to derive the Schrödinger's equation from the same covariance principle [Ste06].

In this paper, we shall see how, this general covariance principle can also be extended to the case of an interface. That is, a singular situation where the metric is not anymore smooth, but has a discontinuity along a hypersurface. This is interpreted as a refraction/reflexion problem, as we shall see. And we shall get then, as an application of this extended general covariance principle, the Snell-Descartes law of reflection and refraction of light.

THANks. - I am thankful to the referee for the careful reading of the manuscript, and for his remarks which contributed to enhance the content of this paper. 


\section{The General Covariance Principle with interface}

In the following we shall consider the following situation, described by Fig. 1. A manifold $M$ is split in two parts $M_{1}$ and $M_{2}$ by an embedded hypersurface $\Sigma$. We can consider that $\mathrm{M}_{1}$ and $\mathrm{M}_{2}$ are two manifolds with a shared boundary $\Sigma$, but their union is the smooth manifold without boundary M:

$$
\mathrm{M}_{1} \cap \mathrm{M}_{2}=\Sigma \text { and } \mathrm{M}_{1} \cup \mathrm{M}_{2}=\mathrm{M} \text {. }
$$

Next, on each part $\mathrm{M}_{1}$ and $\mathrm{M}_{2}$ we define two smooth Riemannian (or PseudoRiemannian) metrics $g_{1}$ and $g_{2}$. That means precisely that $g_{1}$ is the restriction to $\mathrm{M}_{1}$ of a metric defined on a small open neighborhood of $\mathrm{M}_{1}$, idem for $g_{2}$ with $\mathrm{M}_{2}$. The two metrics have then a limit on $\Sigma$ which may not coincide. We shall denote by $g=\left(g_{1}, g_{2}\right)$ this metric on $\mathrm{M}$, having a discontinuity on $\Sigma$.

Next, we introduce the subgroup of compact supported diffeomorphisms of M preserving the hypersurface $\Sigma$,

$$
\operatorname{Diff}_{\mathrm{cpct}}(\mathrm{M}, \Sigma)=\left\{\varphi \in \operatorname{Diff}_{\mathrm{cpct}}(\mathrm{M}) \mid \varphi(\Sigma)=\Sigma\right\} \text {. }
$$

Actually we want $\varphi$ to preserve separately the two parts $M_{1}$ and $M_{2}$. That is, $\varphi \in \operatorname{Diff}_{\text {cpct }}(\mathrm{M}), \varphi\left\lceil\mathrm{M}_{1} \in \operatorname{Diff}_{\mathrm{cpct}}\left(\mathrm{M}_{1}\right), \varphi\left\lceil\mathrm{M}_{2} \in \operatorname{Diff}_{\mathrm{cpct}}\left(\mathrm{M}_{2}\right)\right.\right.$, and of course $\varphi\left\lceil\Sigma \in \operatorname{Diff}_{\text {cpct }}(\Sigma)\right.$. To say that $\varphi$ preserves $\Sigma$ and is connected to the identity would be sufficient.

Now, we introduce the test tensors for the distribution tensors relative to this configuration. Let $g=\left(g_{1}, g_{2}\right)$ be a metric on $\mathrm{M}=\left(\mathrm{M}_{1}, \mathrm{M}_{2}\right)$ as described above and let $s \mapsto g_{s}=\left(g_{1, s}, g_{2, s}\right)$ be two smooth paths in the set of metrics, pointed at $g$, that is, $g_{0}=g$. We shall denote $\delta g=\left(\delta g_{1}, \delta g_{2}\right)$ the variation of $g_{s}$ at $s=0$,

$$
\delta g_{1}=\left.\frac{\partial g_{1, s}}{\partial s}\right|_{s=0} \quad \text { and } \quad \delta g_{2}=\left.\frac{\partial g_{2, s}}{\partial s}\right|_{s=0} .
$$

Therefore, an infinitesimal diffeomorphism which preserves the figure gives a variation of $g$ which is a Lie derivative:

$$
\delta_{\mathrm{L}} g=\left(\delta_{\mathrm{L}} g_{1}, \delta_{\mathrm{L}} g_{2}\right) \quad \text { with } \quad \delta_{\mathrm{L}} g_{i}=\left.\frac{\partial\left(e^{s \xi}\right)^{*}\left(g_{i}\right)}{\partial s}\right|_{s=0},
$$

where $\xi$ is a smooth compact supported vector field on $\mathrm{M}$ such that $\xi(x) \in$ $\mathrm{T}_{x} \Sigma$ for all $x \in \Sigma$. Its exponential $e^{s} \xi$ belongs to $\operatorname{Diff}_{\text {cpct }}(\Sigma)$. We have then

$$
\left(\delta_{\mathrm{L}} g_{i}\right)_{\mu \nu}=\hat{\partial}_{i, \mu} \xi_{\nu}+\hat{\partial}_{i, \nu} \xi_{\mu},
$$


where $\hat{\partial}_{i}$ is the covariant derivative with respect to $g_{i}$. Let us come back to the tensor distribution: according to what was said until now, by linearity $\mathscr{T}$ splits in the sum of two tensors, each relative to a part of $\mathrm{M}$. That is,

$$
\mathscr{T}(\delta g)=\mathscr{T}_{1}\left(\delta g_{1}\right)+\mathscr{T}_{2}\left(\delta g_{2}\right) .
$$

The tensor distribution $\mathscr{T}_{1}$ is equal to $\mathscr{T}(\delta g)$ for $\delta g_{2}=0$, as well for $\mathscr{T}_{2}$ with $\delta g_{1}=0$. Therefore, for $\mathscr{T}$ to be Eulerian means:

$$
\mathscr{T}\left(\delta_{\mathrm{L}} g\right)=0 \Leftrightarrow \mathscr{T}_{1}\left(\delta_{\mathrm{L}} g_{1}\right)+\mathscr{T}_{2}\left(\delta_{\mathrm{L}} g_{2}\right)=0
$$

\section{Crossing the Border}

As a first case, let us consider a continuous medium described by a tensor $\mathrm{T}^{\mu \nu}$, extended on $M_{1}$ and $M_{2}$. That is,

$$
\mathscr{T}(\delta g)=\frac{1}{2} \int_{M} \mathrm{~T}_{1}^{\mu \nu} \delta g_{1, \mu \nu} \operatorname{vol}+\frac{1}{2} \int_{\mathrm{M}} \mathrm{T}_{2}^{\mu \nu} \delta g_{2, \mu \nu} \text { vol, }
$$

with $\mathrm{T}_{a}=\mathrm{T} \uparrow \mathrm{M}_{a}$ and $\delta g_{a}=\delta g \uparrow \mathrm{M}_{a}$. Considering a point $x \in \mathrm{M}$ but not on $\Sigma$ we can find a vector field $\xi$ with support contained in $\mathrm{M}_{1}$ or $\mathrm{M}_{2}$, but avoiding $\Sigma$. Thus, the condition established above applies and we have $\hat{\operatorname{divT}}_{a}=0$ on $\mathrm{M}_{a}-\Sigma$, and by continuity, on $\mathrm{M}_{a}$ including $\Sigma$. Therefore, the Euler equations are still satisfied for each side of the continuous medium.

$$
\hat{\partial}_{\mu} \mathrm{T}_{1}^{\mu \nu}=0 \text { and } \hat{\partial}_{\mu} \mathrm{T}_{2}^{\mu \nu}=0 .
$$

Next, let us consider a vector field $\xi$ with compact support, extending over the two parts $\mathrm{M}_{1}$ and $\mathrm{M}_{2}$. The Eulerian condition writes:

$$
0=\int_{\mathrm{M}_{1}} \mathrm{~T}_{1}^{\mu \nu} \hat{\partial}_{\mu} \xi_{\nu} \operatorname{vol}_{1}+\int_{\mathrm{M}_{2}} \mathrm{~T}_{2}^{\mu \nu} \hat{\partial}_{\mu} \xi_{\nu} \operatorname{vol}_{2} .
$$

Integrating by parts, and after applying Euler equations, we get:

$$
\int_{\mathrm{M}_{1}} \hat{\partial}_{\mu}\left(\mathrm{T}_{1}^{\mu \nu} \xi_{\nu}\right) \operatorname{vol}_{1}+\int_{\mathrm{M}_{2}} \hat{\partial}_{\mu}\left(\mathrm{T}_{2}^{\mu \nu} \xi_{\nu}\right) \operatorname{vol}_{2}=0
$$

Introducing the vector $\mathrm{T}_{a}(\xi)$ defined by its components $\mathrm{T}_{a, \nu}^{\mu \nu} \xi^{\nu}=\mathrm{T}_{a}^{\mu \nu} \xi_{\nu}$, with $\mathrm{T}_{a, \nu}^{\mu}=g_{a, \nu \lambda} \mathrm{T}_{a}^{\lambda \mu}$, we get

$$
\int_{\mathrm{M}_{1}} \hat{\operatorname{div}}\left(\mathrm{T}_{1}(\xi)\right) \operatorname{vol}_{1}+\int_{\mathrm{M}_{2}} \hat{\operatorname{div}}\left(\mathrm{T}_{2}(\xi)\right) \operatorname{vol}_{2}=0 .
$$


That is, using the identity ${ }^{2} \hat{\operatorname{div}}(\theta) \operatorname{vol}=d[\operatorname{vol}(\theta)]$ and Stoke's theorem,

$$
\begin{aligned}
0 & =\int_{\mathrm{M}_{1}} d\left[\operatorname{vol}_{1}\left(\mathrm{~T}_{1}(\xi)\right)\right]+\int_{\mathrm{M}_{2}} d\left[\operatorname{vol}_{2}\left(\mathrm{~T}_{2}(\xi)\right)\right] \\
& =\int_{\partial \mathrm{M}_{1}} \operatorname{vol}_{1}\left(\mathrm{~T}_{1}(\xi)\right)+\int_{\partial \mathrm{M}_{2}} \operatorname{vol}_{2}\left(\mathrm{~T}_{2}(\xi)\right)
\end{aligned}
$$

Then, we can orient $\mathrm{M}$ and $\Sigma$ such that: $\partial \mathrm{M}_{1}=\Sigma$, and then $\partial \mathrm{M}_{2}=-\Sigma$. Hence,

$$
\int_{\Sigma} \operatorname{vol}_{1}\left(\mathrm{~T}_{1}(\xi)\right)=\int_{\Sigma} \operatorname{vol}_{2}\left(\mathrm{~T}_{2}(\xi)\right),
$$

which implies and is equivalent to the condition:

$$
\operatorname{vol}_{1}\left(\mathrm{~T}_{1}(\delta x)\right)\left\lceil\Sigma=\operatorname{vol}_{2}\left(\mathrm{~T}_{2}(\delta x)\right) \uparrow \Sigma .\right.
$$

for all $x \in \Sigma$ and $\delta x \in \mathrm{T}_{x} \Sigma$. Hence, the tensor $\mathrm{T}=\left(\mathrm{T}_{1}, \mathrm{~T}_{2}\right)$ is Eulerian in presence of the interface $\Sigma$ if the Euler equations above are satisfied, together with this boundary condition.

Let $\mathrm{J}$ be a vector transverse to $\Sigma$ at the point $x$, complete with a basis $\mathscr{B}=$ $\left(e_{1}, \ldots, e_{m}\right)$ of $\mathrm{T}_{x} \Sigma$ to get a basis $(\mathrm{J}, \mathscr{B})$ of $\mathrm{T}_{x} \mathrm{M}$. In that basis $\operatorname{vol}_{1}$ and $\operatorname{vol}_{2}$ write

$$
\sqrt{\left|\operatorname{det}\left(\mathrm{G}_{1}\right)\right|} \text { det and } \sqrt{\left|\operatorname{det}\left(\mathrm{G}_{2}\right)\right|} \text { det. }
$$

where $\mathrm{G}_{1}, \mathrm{G}_{2}$ are the Gramm matrices of the metrics $g_{1}, g_{2}$. Let $\left(\mathrm{J}^{*}, \mathscr{B}^{*}\right)$ be the dual basis of $(\mathrm{J}, \mathscr{B})$, with $\mathscr{B}^{*}=\left(e_{1}^{*}, \ldots, e_{m}^{*}\right)$. That is, $\mathrm{J}^{*} \mathrm{~J}=1, \mathrm{~J}^{*} e_{i}=e_{i}^{*} \mathrm{~J}=$ $0, e_{i}^{*} e_{j}=\delta_{i j}$. We introduce the projectors on $\mathbf{R J}$ and $\mathrm{T}_{x} \Sigma$, associated with the basis $(\mathrm{J}, \mathscr{B})$ :

$$
\mathrm{JJ}^{*} \text { and } \mathscr{B} \mathscr{B}^{*}=\sum_{i=1}^{m} e_{i} e_{i}^{*},
$$

where we identified the vectors with their matrix representations. Then, the condition above writes, where the letters $\mathrm{T}_{a}$ denote the matrices associates with the tensors denoted by the same letter:

$$
\mathrm{J}^{*}\left[\sqrt{\left|\operatorname{det}\left(\mathrm{G}_{1}\right)\right|} \mathrm{T}_{1}-\sqrt{\left|\operatorname{det}\left(\mathrm{G}_{2}\right)\right|} \mathrm{T}_{2}\right] \mathscr{B} \mathscr{B}^{*}=0 .
$$

Now, in the framework of relativity (special or general), this condition must be specified for each kind of medium: elastic, fluid or dust. We leave the discussion of these cases for future work. But note already that the case of dust, for which particles follow geodesics, leads to the special treatment that we shall address in the following part.

\footnotetext{
${ }^{2}$ The notation $\operatorname{vol}(\theta)$ denotes the contraction of the form vol by the vector $\theta$.
} 


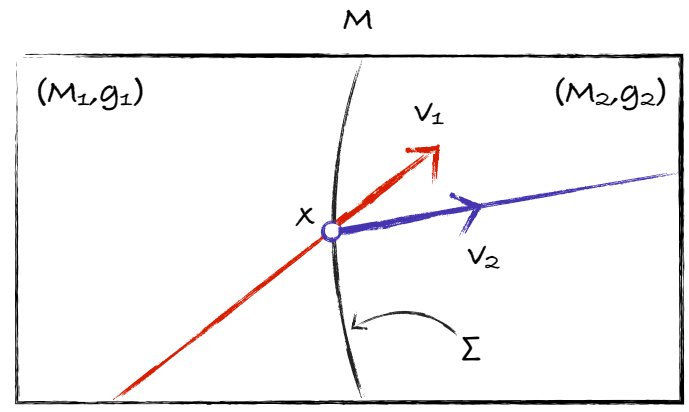

Figure 1. Refraction of Geodesics

The Broken Geodesics

We consider now a curve $\gamma$ in $\mathrm{M}$ that cuts $\Sigma$ at some point $x$ for $t=0$, and let $\gamma_{1}$ and $\gamma_{2}$ be the two pieces of $\gamma$,

$\left.\left.\gamma_{1}:\right]-\infty, 0\right] \rightarrow M_{1}$ and $\gamma_{2}:\left[0,+\infty\left[\rightarrow M_{2}\right.\right.$, with $\quad \gamma_{1}(0)=\gamma_{2}(0)=x$

Let $\mathscr{T}$ be a tensor distribution supported by $\gamma$,

$$
\mathscr{T}(\delta g)=\frac{1}{2} \int_{-\infty}^{0} \mathrm{~T}_{1}^{\mu \nu} \delta g_{1, \mu \nu} d t+\frac{1}{2} \int_{0}^{+\infty} \mathrm{T}_{2}^{\mu \nu} \delta g_{2, \mu \nu} d t .
$$

Solving the Eulerian condition $\mathscr{T}\left(\delta_{\mathrm{L}} g\right)=0$, by considering a vector field $\xi$ whose supports lie first anywhere in $M_{1}$, then in $M_{2}$ we get, thanks to Souriau's computation, that $\gamma_{1}$ and $\gamma_{2}$ are geodesic for the respective metric and also that:

$$
\mathscr{T}(\delta g)=\frac{k_{1}}{2} \int_{-\infty}^{0} \frac{d x^{\mu}}{d t} \frac{d x^{\nu}}{d t} \delta g_{\mu \nu} d t+\frac{k_{2}}{2} \int_{0}^{+\infty} \frac{d x^{\mu}}{d t} \frac{d x^{\nu}}{d t} \delta g_{\mu \nu} d t .
$$

Where $k_{1}$ and $k_{2}$ are a priori two arbitrary real constants. Applied to $\delta_{\mathrm{L}} g$ for a compact supported vector field $\xi$ on $\mathrm{M}$, tangent to $\Sigma$ on $\Sigma$, one gets, by regroupings terms, using the fact that the curves are geodesic, and integrating by parts:

$$
\begin{aligned}
0 & =k_{1} \int_{-\infty}^{0} \frac{d x^{\mu}}{d t} \frac{d x^{\nu}}{d t} \hat{\partial}_{1, \nu} \xi_{\mu}, d t+k_{2} \int_{0}^{+\infty} \frac{d x^{\mu}}{d t} \frac{d x^{\nu}}{d t} \hat{\partial}_{2, \nu} \xi_{\mu}, d t \\
& =k_{1} \int_{-\infty}^{0} \frac{\hat{\partial}_{1} \xi_{\mu}}{\partial x^{\nu}} \frac{d x^{\nu}}{d t} \frac{d x^{\mu}}{d t} d t+k_{2} \int_{0}^{+\infty} \frac{\hat{\partial}_{2} \xi_{\mu}}{\partial x^{\nu}} \frac{d x^{\nu}}{d t} \frac{d x^{\mu}}{d t} d t \\
& =k_{1} \int_{-\infty}^{0} \frac{\hat{d}_{1} \xi_{\mu}}{d t} \frac{d x^{\mu}}{d t} d t+k_{2} \int_{0}^{+\infty} \frac{\hat{d}_{2} \xi_{\mu}}{d t} \frac{d x^{\mu}}{d t} d t
\end{aligned}
$$




$$
\begin{aligned}
& =k_{1} \int_{-\infty}^{0} \frac{\hat{d}_{1}}{d t}\left[\xi_{\mu} \frac{d x^{\mu}}{d t}\right] d t+k_{2} \int_{0}^{+\infty} \frac{\hat{d}_{2}}{d t}\left[\xi_{\mu} \frac{d x^{\mu}}{d t}\right] d t \\
& =k_{1} \xi(x)_{\mu} \lim _{t \rightarrow 0^{-}} \frac{d x^{\mu}}{d t}-k_{2} \xi(x)_{\mu} \lim _{t \rightarrow 0^{+}} \frac{d x^{\mu}}{d t} .
\end{aligned}
$$

Then, denoting by $\mathbf{v}_{1}$ and $\mathbf{v}_{2}$ the limits of the velocities

$$
\mathbf{v}_{1}=\lim _{t \rightarrow 0^{-}} \dot{\gamma}_{1}(t) \quad \text { and } \quad \mathbf{v}_{2}=\lim _{t \rightarrow 0^{+}} \dot{\gamma}_{2}(t)
$$

we get the Eulerian condition for the tensor distribution $\mathscr{T}$ at the point $x \in \Sigma$ which writes

$$
k_{1} g_{1}\left(\mathbf{v}_{1}, \delta x\right)=k_{2} g_{2}\left(\mathbf{v}_{2}, \delta x\right) \text { for all } \delta x \in \mathrm{T}_{x}(\Sigma) .
$$

We get then a general condition which must be satisfied by the two geodesics $\gamma_{1}$ and $\gamma_{2}$ at the interface, to define an Eulerian distribution. But the system is underdetermined due to the arbitrary choice of constants $k_{1}$ and $k_{2}$. We can diminish the arbitrary by considering the symplectic structure of the spaces of geodesics.

REMARK. - The case above admits a solution when there is no part 2, and just the curve $\gamma_{1}$. In that case the equation $(\boldsymbol{\phi})$ becomes $g_{1}\left(\mathbf{v}_{1}, \delta x\right)=0$ for all $\delta x \in \mathrm{T}_{x}(\Sigma)$, that is,

$$
\mathbf{v}_{1} \perp \Sigma .
$$

Hence, the Eulerian distributions are the geodesics which end orthogonally at the intersection with the interface. It is the case in the Poincare half-plan, for example. That has been observed in real world, with light rays in a saline solution for which the concentration tends to infinity near the border.

\section{The Symplectic Scattering}

We shall use now the symplectic structure of the space of geodesics and we shall request the distribution $\mathscr{T}$ to be a symplectic scattering $\mathscr{T}_{1} \rightarrow \mathscr{T}_{2}$, in order to lower the degree of indetermination in the equation $(\boldsymbol{P})$.

Let us recall now that the space $\mathfrak{Z}$ of geodesics curves in a Riemannian (or Pseudo-Riemannian) manifold $(\mathrm{M}, g$ ) has a canonical symplectic structure. It is the projection of the exterior derivative $d \varpi$ of the Cartan 1-form $\varpi$ defined on $\mathrm{Y}=\mathbf{R} \times(\mathrm{TM}-\mathrm{M})$ by

$$
\varpi(\delta y)=k g(\mathbf{v}, \delta x)-\frac{k}{2} g(\mathbf{v}, \mathbf{v}) \delta t,
$$


where $y=(t, x, \mathbf{v}) \in \mathrm{Y}$ and $\delta y \in \mathrm{T}_{y} \mathrm{Y}$. Let $\mathfrak{Z}_{1}$ and $\mathfrak{Z}_{2}$ be the space of geodesics in $\left(\mathrm{M}_{1}, g_{1}\right)$ and $\left(\mathrm{M}_{2}, g_{2}\right)$. In the two cases a parametrized geodesic that cut (transversally) the interface $\Sigma$ is completely determined by $(t, x, \mathbf{v})$, where $x \in \Sigma$ is the intersection of the geodesic with the interface, $t \in \mathbf{R}$ is the time of the impact and $\mathbf{v} \in \mathrm{T}_{x} \mathrm{M}$ is the velocity of the geodesic at the point $x$. This defined two open subsets $\mathfrak{Z}_{1}^{\star} \subset \mathfrak{Z}_{1}$ and $\mathfrak{Z}_{2}^{\star} \subset \mathfrak{Z}_{2}$. These two subspaces are equipped naturally with the restriction of their Cartan form $\varpi_{1}$ and $\varpi_{2}$, as stated above.

Because of the indetermination we have no clear map $\mathscr{T}_{1} \rightarrow \mathscr{T}_{2}$, but a relation $\mathscr{R}$ that we can describe by its graph in $\mathfrak{Z}_{1}^{\star} \times \mathfrak{Z}_{2}^{\star}$. Let $y_{1}=\left(t_{1}, x_{1}, \mathbf{v}_{1}\right)$ and $y_{2}=\left(t_{2}, x_{2}, \mathbf{v}_{2}\right)$ represent two geodesics in each of these spaces:

$$
y_{1} \mathscr{R} y_{2} \Leftrightarrow\left\{\begin{array}{l}
t_{1}=t_{2}=t, \quad x_{1}=x_{2}=x \\
\text { and } \\
k_{1} g_{1}\left(\mathbf{v}_{1}, \delta x\right)=k_{2} g_{2}\left(\mathbf{v}_{2}, \delta x\right) \quad \text { for all } \delta x \in \mathrm{T}_{x} \Sigma .
\end{array}\right.
$$

The first condition translates the fact that the two geodesics cut the interface at the same point at the same time, and the second condition describes the Eulerian nature of the scattering, expressed in ( $)$.

Actually, we shall ask $\mathscr{R}$ to preserve the Cartan form. It is a stronger request but usual in this kind of problems. Let us precise that, for the relation $\mathscr{R}$, to preserve the Cartan form means that the form $\varpi_{1} \ominus \varpi_{2}$, defined on $\mathfrak{Z}_{1}^{\star} \times \mathfrak{Z}_{2}^{\star}$, vanishes on $\mathscr{R}$. That is, for all $y \in \mathscr{R}$, if $\delta y \in \mathrm{T}_{y} \mathscr{R}$ then $\varpi_{1} \ominus \varpi_{2}(\delta y)=0$. But

$$
\begin{aligned}
\varpi_{1} \ominus \varpi_{2}(\delta y) & =k_{1} g_{1}\left(\mathbf{v}_{1}, \delta x\right)-\frac{k_{1}}{2} g_{1}\left(\mathbf{v}_{1}, \mathbf{v}_{1}\right) \delta t \\
& -k_{2} g_{2}\left(\mathbf{v}_{2}, \delta x\right)+\frac{k_{2}}{2} g_{2}\left(\mathbf{v}_{2}, \mathbf{v}_{2}\right) \delta t
\end{aligned}
$$

Therefore, $\mathscr{R}$ preserves the Cartan form between the two spaces of geodesics if and only if

$$
k_{1} g_{1}\left(\mathbf{v}_{1}, \mathbf{v}_{1}\right)=k_{2} g_{2}\left(\mathbf{v}_{2}, \mathbf{v}_{2}\right) .
$$

We notice here that $k_{1} g_{1}\left(\mathbf{v}_{1}, \mathbf{v}_{1}\right) / 2$ is the moment of the symmetry group of time translation $(t, x, \mathbf{v}) \mapsto(t+e, x, \mathbf{v})$, for all $e \in \mathbf{R}$. And preserving the Cartan form is equivalent to the preservation of the energy-moment when crossing the interface (which is not surprising). It describes a conservative - i.e. non-dissipative - process, which is the rule in symplectic mechanics. 


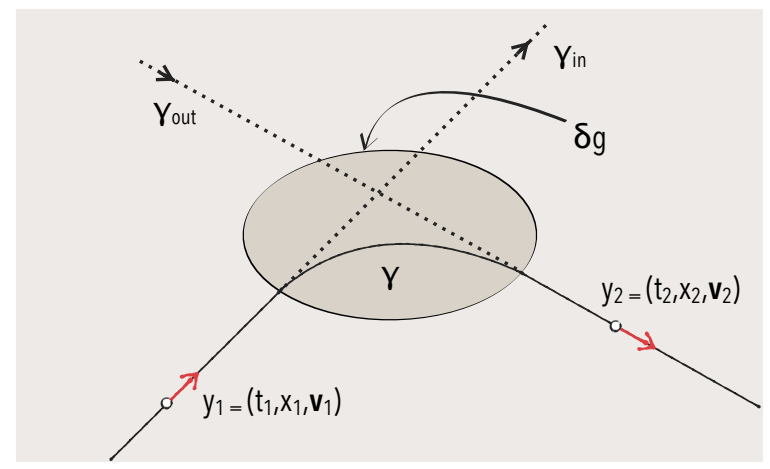

Figure 2. A Metric Blob

Thus, the symplectic framework decreases the level of indetermination in the scattering process, but it remains the arbitrary of the ratio $k_{2} / k_{1}$ which maybe lifted by a state function proper to the system considered.

We shall see that, for geometric optics, the physical context lifts indeed the indetermination.

Note 1. - This construction also answers the question of reflexion, when $\gamma_{2}$ lies in $\mathrm{M}_{1}$ too. In this case $k_{1}=k_{2}$ and the symplectic condition states that $\mathbf{v}_{1}$ and $\mathbf{v}_{2}$ have the same norm relatively to $g_{1}$, which solves the issue without other considerations.

Note 2. - The requirement of symplectic scattering is justified by the following situation. Consider a manifold $M$, equipped with some metric $g$. Its space of parametrized geodesics $\mathscr{G}$ is a symplectic manifold for the pushforward $\omega$ of the differential $d \varpi$ of the Cartan form. Now consider a perturbation $g^{\prime}$ of $g$, such that the two metrics differ only on a compact $\mathrm{K} \subset \mathrm{M}$. For the sake of simplicity, we assume that every geodesic for $g^{\prime}$ leaves the compact $\mathrm{K}$ in the past and the future. The set of parametrized geodesics $\mathscr{G}^{\prime}$ of $\left(\mathrm{M}, g^{\prime}\right)$ is symplectic for $\omega^{\prime}$. Consider a geodesic $\gamma \in \mathscr{G}^{\prime}$, before entering in the compact $\mathrm{K}$ it defines a geodesic $\gamma_{\text {in }} \in \mathscr{G}$, and after exiting the compact it defines a geodesic $\gamma_{\text {out }} \in \mathscr{G}$, as it is described in Fig. 2. That defines a scattering $\varphi: \gamma_{\text {in }} \mapsto \gamma_{\text {out }}$ on a subset of $\mathscr{G}$, the geodesics crossing the compact K. Let us check that $\varphi$ is a local symplectomorphism. Let $\gamma \in \mathscr{G}^{\prime}$, and $\delta \gamma$ and $\delta \gamma^{\prime}$ be two tangents vectors at $\gamma \in \mathscr{G}$. Let $y=(t, x, \mathbf{v})$ be an initial condition for $\gamma$, let $\delta y=(\delta t, \delta x, \delta \mathbf{v})$ and $\delta^{\prime} y=\left(\delta^{\prime} t, \delta^{\prime} x, \delta^{\prime} \mathbf{v}\right)$ be two tangent vectors projecting on $\delta \gamma$ and $\delta^{\prime} \gamma$. Then, $\omega^{\prime}\left(\delta \gamma, \delta \gamma^{\prime}\right)=d \varpi^{\prime}\left(\delta y, \delta^{\prime} y\right)$. But we can evaluate $d \varpi^{\prime}\left(\delta y, \delta^{\prime} y\right)$ at a point $y_{1}=\left(t_{1}, x_{1}, \mathbf{v}_{1}\right)$ before $\gamma$ entering the compact $\mathrm{K}$. In this case $\omega^{\prime}\left(\delta \gamma, \delta \gamma^{\prime}\right)=d \varpi^{\prime}\left(\delta y_{1}, \delta^{\prime} y_{1}\right)=d \varpi\left(\delta y_{1}, \delta^{\prime} y_{1}\right)=\omega\left(\delta \gamma_{\text {in }}, \delta^{\prime} \gamma_{\text {in }}\right)$. And we can also evaluate $d \varpi^{\prime}\left(\delta y, \delta^{\prime} y\right)$ at a point $y_{2}=\left(t_{2}, x_{2}, \mathbf{v}_{2}\right)$ after $\gamma$ leaving 
the compact K. in this case $\omega^{\prime}\left(\delta \gamma, \delta \gamma^{\prime}\right)=d \varpi^{\prime}\left(\delta y_{2}, \delta^{\prime} y_{2}\right)=d \varpi\left(\delta y_{2}, \delta^{\prime} y_{2}\right)=$ $\omega\left(\delta \gamma_{\text {out }}, \delta^{\prime} \gamma_{\text {out }}\right)$. Therefore, $\omega\left(\delta \gamma_{\text {out }}, \delta^{\prime} \gamma_{\text {out }}\right)=\omega\left(\delta \gamma_{\text {in }}, \delta^{\prime} \gamma_{\text {in }}\right)$, that is $\varphi^{*}(\omega)=\omega$. The scattering $\varphi: \gamma_{\text {in }} \mapsto \gamma_{\text {out }}$, which is bijective (by $t \mapsto-t$ ), is then a symplectomorphism. Hence, assuming that the scattering of geodesics by an interface - dividing two different media - is symplectic, is completely legitimate.

\section{The Case of Light}

Let us consider now the special case of light rays propagating in two homogeneous media with different indices $n_{1}$ and $n_{2}$, separated by the hypersurface $\Sigma$. We shall see that the particular context of geometric optics will achieve to lift the indetermination in the rule $(\boldsymbol{\rho})$, with the condition of symplectic scattering ( $\mathbf{p})$. Let $v_{1}$ and $v_{2}$ be the speed of light in the two different media. Then,

$$
g_{1}=n_{1}^{2} g_{0} \quad \text { and } g_{2}=n_{2}^{2} g_{0} \quad \text { with } \quad n_{1}=\frac{c}{v_{1}} \quad \text { and } \quad n_{2}=\frac{c}{v_{2}},
$$

where $g_{0}$ is an ambient smooth metric on $\mathrm{M}$, the vacuum metric. Assuming $\mathrm{M} \subset \mathbf{R}^{3}$, we chose $g_{0}$ to be the standard scalar product, and we note as usual $g_{0}(\mathbf{u}, \mathbf{v})=\mathbf{u} \cdot \mathbf{v}$ and $g_{0}(\mathbf{v}, \mathbf{v})=\|\mathbf{v}\|^{2}$. Let us recall that we defined the color coefficients as

$$
k_{1}=\frac{b v_{1}}{c^{2}} \text { and } k_{2}=\frac{b v_{2}}{c^{2}}
$$

and let,

$$
\mathbf{v}_{1}=v_{1} \mathbf{u}_{1} \quad \text { and } \quad \mathbf{v}_{2}=v_{1} \mathbf{u}_{2} \text { with }\left\|\mathbf{u}_{1}\right\|^{2}=\left\|\mathbf{u}_{2}\right\|^{2}=1
$$

Next, the symplectic condition (⿳⺈⿴囗十) writes

$$
k_{1} n_{1}^{2}\left\|\mathbf{v}_{1}\right\|^{2}=k_{2} n_{2}^{2}\left\|\mathbf{v}_{2}\right\|^{2}, \quad \text { that is, } \quad k_{1} \frac{c^{2}}{v_{1}^{2}} v_{1}^{2}=k_{2} \frac{c^{2}}{v_{2}^{2}} v_{2}^{2} .
$$

Hence,

$$
k_{1}=k_{2} \quad \text { i.e. } \quad v_{1}=v_{2} \text {. }
$$

The frequency of the light ray, its color, does not change when crossing the interface. Now, the covariant condition ( $(\mathbf{)})$ writes, for all $\delta x \in \mathrm{T}_{x} \Sigma$,

$n_{1}^{2} \mathbf{v}_{1} \cdot \delta x=n_{2}^{2} \mathbf{v}_{2} \cdot \delta x \Leftrightarrow \frac{c^{2}}{v_{1}^{2}} v_{1} \mathbf{u}_{1} \cdot \delta x=\frac{c^{2}}{v_{2}^{2}} v_{2} \mathbf{u}_{2} \cdot \delta x \Rightarrow \frac{c}{v_{1}} \mathbf{u}_{1} \cdot \delta x=\frac{c}{v_{2}} \mathbf{u}_{2} \cdot \delta x$.

That is, $\left(n_{1} \mathbf{u}_{1}-n_{2} \mathbf{u}_{2}\right) \cdot \delta x=0$ for all $\delta x \in \mathrm{T}_{x} \Sigma$. And then,

$$
n_{2} \mathbf{u}_{2}=n_{1} \mathbf{u}_{1}+\alpha \mathbf{n}
$$


where $\mathbf{n}$ is the unit normal vector to $\Sigma$ at $x$, and $\alpha$ some number. By decomposing the unit vectors $\mathbf{u}_{1}$ and $\mathbf{u}_{2}$ in an orthonormal basis completing $\mathbf{n}-$ in the plane spanned by $\mathbf{u}_{1}$ and $\mathbf{n}-$ and denoting by $\theta_{i}$ the angles between the $\mathbf{u}_{i}$ and the normal $\mathbf{n}$, we deduce the classical Snell-Descartes law of refraction of light,

$$
n_{1} \sin \left(\theta_{1}\right)=n_{2} \sin \left(\theta_{2}\right)
$$

In Conclusion, the law of refraction of light becomes a consequence of the general covariance principle, conjugated with the symplectic structure of the space of rays. The case of reflexion is also covered and gives as usual $\sin \left(\theta_{1}\right)=$ $\sin \left(\theta_{2}\right)$.

It will be noted again that in these cases, the distribution tensor $\mathscr{T}$ defines a scattering map $\mathscr{T}_{1} \rightarrow \mathscr{T}_{2}$, from the inward geodesic $\gamma_{1}$ to the outward geodesic $\gamma_{2}$.

\section{BEHIND THE SCENES}

It is very intriguing that the evolution of material media, that are generally described by a principle of least action, can be also described by a principle of invariance (actually covariance) by diffeomorphisms. The medium can be elastic, or a particle, or even a light ray refracted by an interface.

There is always some kind of mystery behind the principle of least action, sort of God willing principle. Or the Nature which - by a hidden will decides at each instant what is the best for the system in its immediate future. Every student who has been there once, knows this feeling of some magic that governs the world of matter.

And suddenly, we have the same behavior described just by an invariance principle, where all the magic has disappeared: that material presence and its evolution do not depend on the way they are described, they have an objective nature. And it is this objectivity which is captured by this principle of general covariance: the laws of nature are described by a covector on the space of geometries modulo diffeomorphisms. How is that possible?

Actually, these two principles are not as independent as they seem. The principle of least action and the principle of general covariance are indeed the two faces of the same coin. And to bring that to light, we shall consider the following toy model.

Let $\mathscr{C}$ be a space, we shall call the space of fields. Let $\mathscr{M}$ be a space, we shall call the space of geometries. Here $\mathscr{C}$ and $\mathscr{M}$ will be manifolds. Let $\mathrm{G}$ be a Lie 
group acting on the fields and the geometries. Let $c \in \mathscr{C}, g \in \mathscr{M}$ and $\varphi \in \mathrm{G}$. Let $\varphi_{*}(c)$ and $\varphi_{*}(g)$ be the action of $\varphi$ on $c$ and $g$. Assume that the action of $\mathrm{G}$ on $\mathscr{C}$ is transitive. Let $\mathscr{A}: \mathscr{C} \times \mathscr{M} \rightarrow \mathbf{R}$ be a smooth map we call the action function. Assume that the action is invariant under the diagonal action of $\mathrm{G}$ on $\mathscr{C} \times \mathscr{M}$. That is

$$
\mathscr{A}\left(\varphi_{*}(c), \varphi_{*}(g)\right)=\mathscr{A}(c, g) \text { for all } \varphi \in \mathrm{G} .
$$

Next, consider a 1-parameter subgroup $s \mapsto \varphi_{s}$ in $G$ and let us put

$$
\delta_{\mathrm{L}} c=\left.\frac{\partial \varphi_{s *}(c)}{\partial s}\right|_{s=0} \text { and } \delta_{\mathrm{L}} g=\left.\frac{\partial \varphi_{s}^{*}(g)}{\partial s}\right|_{s=0},
$$

where $\varphi^{*}=\varphi^{-1}$. Therefore, taking the derivative of the invariance identity of the action above, we get that for all infinitesimal action $\delta_{\mathrm{L}}$ of the group $G$ we have

$$
\mathscr{J}\left(\delta_{\mathrm{L}} c\right)-\mathscr{T}\left(\delta_{\mathrm{L}} g\right)=0 \quad \text { with } \quad \mathscr{J}=\frac{\partial \mathscr{A}}{\partial c} \quad \text { and } \quad \mathscr{T}=\frac{\partial \mathscr{A}}{\partial g} .
$$

Thus, obviously, if $\mathscr{T}\left(\delta_{\mathrm{L}} g\right)=0$, then $\mathscr{J}\left(\delta_{\mathrm{L}} c\right)$, and conversely. Hence:

(1) If $c$ is a critical point for $\mathscr{A}_{g}: c \mapsto \mathscr{A}(c, g)$, then $\mathscr{T}\left(\delta_{\mathrm{L}} g\right)=0$ for all $\delta_{\mathrm{L}} g$. That is, $\mathscr{T}$ is Eulerian.

(2) If $\mathscr{T}$ is Eulerian, then $\mathscr{J}\left(\delta_{\mathrm{L}} c\right)=0$ for all $\delta_{\mathrm{L}} c$. But since $\mathrm{G}$ is transitive on $\mathscr{C}$, we get $\mathscr{J}(\delta c)=0$ for any variation $\delta c$. Which means that

$$
\delta \mathscr{A}_{g}=\left.\frac{\partial \mathscr{A}_{g}\left(c_{s}\right)}{\partial s}\right|_{s=0}=0 \text { with } \mathscr{A}_{g}: c \mapsto \mathscr{A}(c, g),
$$

for all smooth path $s \mapsto c_{s}$. Therefore, $c$ is a critical point of the action $\mathscr{A}_{g}$, and a solution of the least action principle for this action.

Of course, for the cases treated in general the space of fields and the space of geometries are not manifolds but infinite dimensional spaces. There is certainly a right way to treat these situations rigorously, from the point of view of diffeology for example. But that is not the point here. The idea here is just to see how the principle of general covariance withdraws the magic from the principle of least action, by giving the main role to the laws of symmetries and the principle of objectivity/relativity which definitely deserve it. At the same time the principle of general covariance is a well founded a priori generalization of the least action principle. 


\section{REFERENCES}

[Sou74] Jean-Marie Souriau. Modèle de particule à spin dans le champ électromagnétique et gravitationnel. Ann. Inst. Henri Poincaré, XX A, 1974.

[Ste06] Shlomo Sternberg. General Covariance and the Passive Equations of Physics. Albert Einstein Memorial Lecture, ISBN 965-208-173-6. The Israel Academy of Sciences and Humanities, Jerusalem 2006.

Patrick Iglesias-Zemmour - Aix Marseille Univ, CNRS, Centrale Marseille, i2M, Marseille, France $-\&-$ Einstein Institute of Mathematics Edmond J. Safra Campus, The Hebrew University of Jerusalem Givat Ram. Jerusalem, 9190401, Israel.

E-mail address: piz@math.huji.ac.il 